\title{
Association analyses of DNA methyltransferase-1 (DNMT1) polymorphisms with systemic lupus erythematosus
}

Received: 24 June 2004 / Accepted: 26 July 2004/ Published online: 16 September 2004

(C) The Japan Society of Human Genetics and Springer-Verlag 2004

\begin{abstract}
The etiology of systemic lupus erythematosus (SLE) is very complex, and genetic factors appear to play a significant role in susceptibility to SLE, in determining the disease expression, and in the autoantibody profiles of individuals with SLE. DNA methyltransferase-1 (DNMT1) is a major enzyme that determines genomic methylation patterns and both maintains methyltransferase and exhibits de novo DNA methylation activity in vivo. In order to clarify the association of DNMT1 polymorphisms with SLE, we scrutinized the genetic polymorphisms in exons and their boundaries of $D N M T 1$, including the $-1,500$ bp promoter region, by direct sequencing in 24 Korean individuals. Twenty-nine sequence variants were identified: two in $5^{\prime}$ UTR, six in exons, and 21 in introns. Eight of these polymorphisms were selected for a larger-scale genotyping $(n=680)$ by considering their allele frequencies, haplotype-tagging status, and linkage disequilibrium coefficiencies (LDs) among polymorphisms. The associations between DNMT1 polymorphisms and the clinical profiles of SLE were analyzed. No significant associations with the risk of SLE were detected. However, further analyses of association with autoantibody production among SLE patients revealed that one nonsynonymous SNP, $+14463 \mathrm{G}>\mathrm{C}(\mathrm{V} 120 \mathrm{~L})$ in exon 4 , was weakly associated with an increased risk of antiLa antibody production $(P=0.04)$, although the significance could not be retained after correction of multiple tests. The DNMTI variations and haplotypes clarified in
\end{abstract}

\footnotetext{
B. L. Park $\cdot$ L. H. Kim $\cdot$ H. D. Shin

Department of Genetic Epidemiology, SNP Genetics, Inc., 11th Floor, MaeHun B/D, 13 Chongro 4 Ga, Chongro-Gu, Seoul, 110-834, South Korea

Y. W. Park · W. S. Uhm · S.-C. Bae $(\bowtie)$

Department of Internal Medicine, Division of Rheumatology, The Hospital for Rheumatic Diseases,

Hanyang University, 17 Haengdang-Dong,

Seongdong-Gu, Seoul, 133-792, South Korea

E-mail: scbae@hanyang.ac.kr

Tel.: + 82-2-22909203

Fax: $+82-2-22988231$
}

this study would provide valuable information for future genetic studies of other autoimmune diseases.

Keywords DNA methyltransferase-1 Polymorphism · Haplotype $\cdot$ Systemic lupus erythematosus . Autoantibody

\section{Introduction}

Systemic lupus erythematosus (SLE) is characterized by the accelerated apoptosis of peripheral lymphocytes and the impaired clearance of apoptotic cells. The combination of these defects results in the release of increased amounts of nucleosomes with abnormally methylated and GC-rich DNA and consequently an autologous stimulation that could bypass tolerance to systemic autoimmune diseases (Huck et al. 1999). The etiology of SLE is very complex, involving both environmental and genetic factors and probably also a synergistic relationship between these factors. Genetic factors are likely to play a significant role in susceptibility to SLE, in determining the disease expression, and in the autoantibody profiles of individuals with SLE (Wakeland et al. 2001). It was recently shown that autoantibodies are associated with SLE showing various clinical manifestations. The appearance of autoantibodies in patients with SLE tends to follow the progressive accumulation of specific autoantibodies before the onset of SLE (Arbuckle et al. 2003; Jacob and Viard 1992; Richardson et al. 1990).

DNA methyltransferase-1 (DNMT1, MIM 126375), located on chromosome 19p13.3-p13.2 with total size $\sim 62 \mathrm{~Kb}$, is a major enzyme that determines genomic methylation patterns and is the best-understood enzyme among three families of DNA (cytosine-5) methyltransferases: DNMT1, DNMT2, DNMT3a, and DNMT3b. The enzyme encoded by DNMT1 functions primarily in the maintenance of methyltransferase, which transfers methyl groups to cytosine in hemimethylated $\mathrm{CpG}$ sites after DNA replication, and it also 
has a role in gene silencing (Bestor 1992; Okano et al. 1998). DNA methylation plays important roles in the modulation chromatin structure, transcriptional regulation, and genomic stability, and has also been shown to be essential for mammalian development (Jones and Takai 2001; Lei et al. 1996; Li et al. 1992). DNMT1 is a major enzyme that determines genomic methylation patterns, functions, maintains methyltransferase, and has a role in gene silencing (Bestor 1992; Okano et al. 1998). DNMT1 also targets replication foci by binding to proliferating cell nuclear antigens and possesses de novo DNA methylation activity in vivo (Chuang et al. 1997; Robertson 2001). Mutations in coding regions of the DNMT1 gene have been analyzed in subjects with cancerous and noncancerous tissues. Two mutations were reported in a colorectal cancer: (1) a one-base deletion in exon 23, resulting in the deletion of the whole catalytic domain, and (2) a point mutation in exon 35, resulted in an amino acid substitution (Tyr to Cys) in the catalytic domain. Inactivation of DNMT1 due to two mutations might be a rare event during human carcinogenesis although mutational inactivation of DNMT1 potentially causes a genome-wide alteration of the DNA methylation status (Kanai et al. 2003).

Despite the functional importance of the DNMT1 gene, polymorphisms of DNMT1 have not been fully examined. In an effort to discover polymorphism(s) in genes whose variant(s) might be implicated in clinical manifestations of SLE, we scrutinized the genetic polymorphisms in DNMT1 by direct sequencing. Here we present 29 genetic variants found in DNMT1, among which eight were genotyped on a larger scale $(n=680)$, and the results of association analyses with phenotypes of SLE in the Korean population.

\section{Materials and methods}

\section{Subjects}

A total of 350 Korean SLE patients who fulfilled 1997 American College of Rheumatology (ACR) criteria of SLE (Hochberg 1997) were consecutively enrolled between September 1998 and February 2002 from the Hospital for Rheumatic Diseases, Hanyang University, Seoul, Korea. The following clinical and laboratory data were obtained: gender, age, ages at first symptom onset and clinical diagnosis, ACR diagnosis, and SLICC/ACR (Systemic Lupus International Collaborating Clinics/ American College of Rheumatology) damage index (Gladman et al. 1996). As a control group, we included 330 healthy ethnically matched subjects in our examination of the genetic association of polymorphisms with susceptibility to SLE and related phenotypes. Testing for antinuclear antibodies (Abs) was carried out by indirect immunofluorescence using IT-1 cells; anti-dsDNA Abs by Crithidia luciliae assay; anti-Sm, anti-SSA (Ro), antiSSB (La), and anti-RNP (ribonuclear protein) Abs by double immunodiffusion.
Sequencing analysis of the DNMT1 gene

We sequenced exons and their boundaries of the DNMT1 gene, including the promoter region $(\sim 1.5 \mathrm{~kb}$ ), to discover genetic variants in 24 Korean DNA samples using a DNA analyzer (ABI PRISM 3700, Applied Biosystems, Foster City, CA, USA). Forty-one primer sets of DNMT1 for the amplification and sequencing analysis were designed based on GenBank sequences (Ref. Genome seq. for DNMT1; NT_011295.10 released in Feb 2004). Information regarding the primers used is available on our Web site (http://www.snp-genetics.com/user/news_content.asp? board_idx =178). Sequence variants were verified by chromatograms.

Genotyping with fluorescence polarization detection

For genotyping of polymorphic sites, amplifying primers and probes were designed for TaqMan (Livak 1999). Information regarding the primers is available on our Web site (http://www.snp-genetics.com/user/news_content.asp?board_idx $=178$ ).

\section{Statistics}

We examined Lewontin's $\mathrm{D}^{\prime}\left(\left|D^{\prime}\right|\right)$ and the linkage disequilibrium coefficient $r^{2}$ between all pairs of biallelic loci (Hedrick and Kumar 2001; Hedrick 1987). Haplotypes of each individual were inferred using the algorithm developed by Stephens et al. (2001) (PHASE), which uses a Bayesian approach incorporating a priori expectations of haplotypic structure based on population genetics and coalescent theory. Genetic effects of inferred haplotypes were analyzed in the same way as polymorphisms. The genotype distributions of $D N M T 1$ polymorphisms and haplotypes between the SLE and normal subjects and among SLE patients were analyzed with logistic regression models whilst controlling for age (continuous variable), gender $($ male $=0$, female $=1$ ), and/or disease duration (continuous variable) as covariates. The common alleles were used as the reference genotype to the heterozygote and homozygote of the minor allele. Probability values of codominant, dominant, and recessive models are also given.

\section{Results and discussion}

To discover polymorphism(s) in $D N M T 1$, we performed direct DNA sequencing in 24 unrelated Korean individuals. We identified 29 sequence variants: two in $5^{\prime}$ UTR, 21 in introns, and six in exons (3 nonsynonymous and 3 synonymous) (Fig. 1a). The two mutations in exons 23 and 35 that had been reported in colorectal cancer patients (Japanese) (Kanai et al. 2003) were not detected in this study. Pair-wise comparisons among 

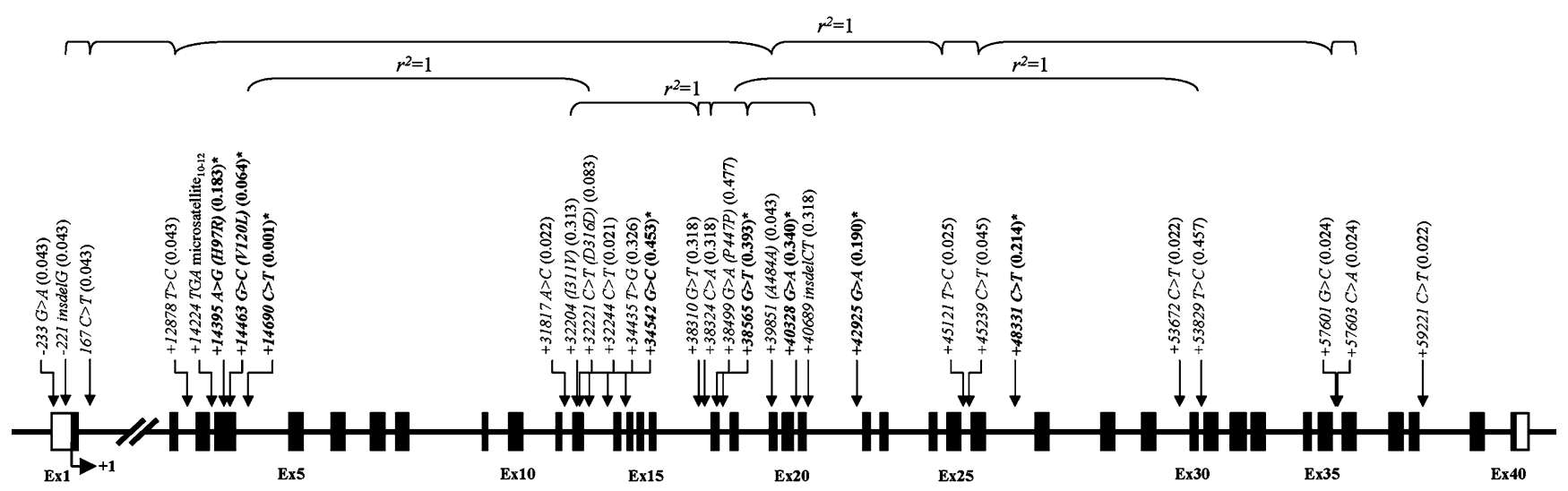

\section{B. Haplotypes of DNMT1 gene}

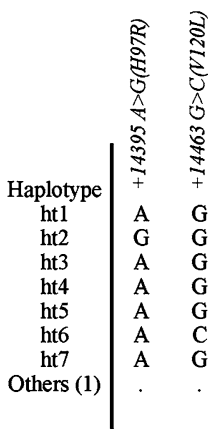

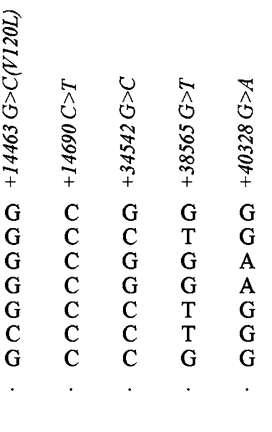

C. LDs among DNMT1 polymorphisms

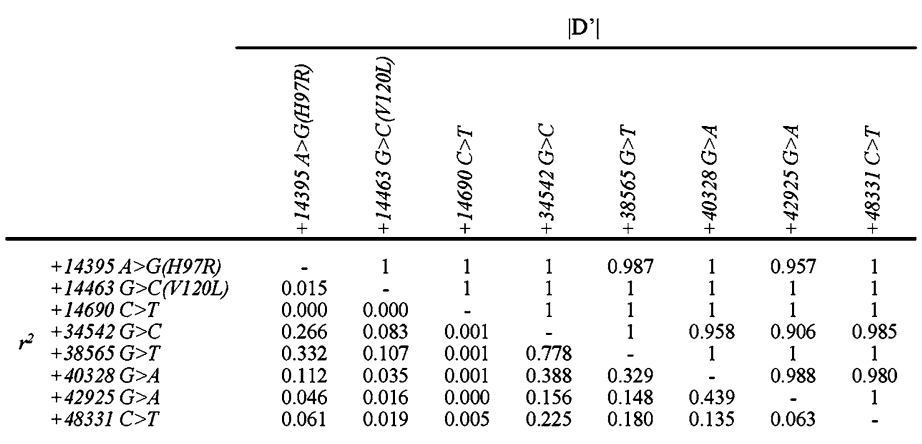

Fig. 1 Gene maps and haplotypes of DNMT1. Coding exons are marked by black blocks and 5' and 3' UTRs by white blocks. The first base of the translational start site is denoted as nucleotide +1 . Asterisks indicate polymorphisms genotyped in a larger population $(n=680)$. The frequencies of polymorphisms not subjected to larger-scale genotyping were based on sequence data $(n=24)$. a Polymorphisms identified in DNMT1 on chromosome 19p13.3p13.2 (Ref. Genome Seq. NT_011295.10). b Haplotypes of $D N M T 1$. Only those with frequencies $\geq 0.05$ are shown. Others (1) contain rare haplotypes: AGCCGAAC, GGCCGGAC, AGCGGAGT, AGCCGGGT, AGTGGGGT, and AGCGGGGC. c Linkage disequilibrium coefficients $\left(\left|D^{\prime}\right|\right.$ and $\left.r^{2}\right)$ among DNMT1 polymorphisms polymorphisms revealed several sets of absolute LDs $\left(\left|D^{\prime}\right|=1\right.$ and $\left.r=1\right)$ and complete LDs $\left(\left|D^{\prime}\right|=1\right.$ and $r^{2} \neq 1$ ) (Fig. 1a,c). Eight polymorphisms in DNMT1 were selected for larger-scale genotyping $(n=680)$ by considering their allele frequencies, haplotype-tagging status, and LDs. SNPs in exons, with higher frequencies and higher haplotype-tagging status, were preferred. The allele frequencies of these eight polymorphisms were $0.182[+14395 \mathrm{~A}>\mathrm{G} \quad(\mathrm{H} 97 \mathrm{R})], 0.064[+14463 \mathrm{G}>\mathrm{C}$ (V120L)], $0.001(+14690 \mathrm{C}>\mathrm{T}), 0.453(+34542 \mathrm{G}>\mathrm{C})$, $0.393(+38565 \mathrm{G}>\mathrm{T}), \quad 0.340 \quad(+40328 \mathrm{G}>\mathrm{A}), \quad 0.190$ $(+42925 \mathrm{G}>\mathrm{A})$, and $0.214(+48331 \mathrm{C}>\mathrm{T})$ (Fig. 1a).
Table 1 Association analysis of $D N M T 1$ polymorphisms and haplotypes with the risk of systemic lupus erythematosus (SLE) in the Korean population $(n=680)$

${ }^{\mathrm{a}} P$ values for logistic analyses of three alternative models (codominant, dominant, and recessive), whilst controlling for age and gender as covariates

\begin{tabular}{|c|c|c|c|c|c|}
\hline \multirow[t]{2}{*}{ Locus } & \multicolumn{2}{|c|}{ Frequency } & \multicolumn{3}{|l|}{$P^{\mathrm{a}}$} \\
\hline & SLE & Normal control & Codominant & Dominant & Recessive \\
\hline + $14395 \mathrm{~A}>\mathrm{G}(\mathrm{H} 97 \mathrm{R})$ & 0.170 & 0.195 & 0.16 & 0.32 & 0.10 \\
\hline$+14463 \mathrm{G}>\mathrm{C}(\mathrm{V} 120 \mathrm{~L})$ & 0.066 & 0.062 & 0.55 & 0.66 & 0.42 \\
\hline$+14690 \mathrm{C}>\mathrm{T}$ & 0.001 & 0.002 & 0.94 & 0.94 & \\
\hline$+34542 \mathrm{G}>\mathrm{C}$ & 0.457 & 0.450 & 0.81 & 0.70 & 0.99 \\
\hline$+38565 \mathrm{G}>\mathrm{T}$ & 0.388 & 0.398 & 0.73 & 0.63 & 0.18 \\
\hline$+40328 \mathrm{G}>\mathrm{A}$ & 0.331 & 0.349 & 0.56 & 0.80 & 0.41 \\
\hline$+42925 \mathrm{G}>\mathrm{A}$ & 0.181 & 0.200 & 0.36 & 0.38 & 0.64 \\
\hline$+48331 \mathrm{C}>\mathrm{T}$ & 0.220 & 0.208 & 0.67 & 0.47 & 0.56 \\
\hline htl & 0.215 & 0.205 & 0.74 & 0.54 & 0.57 \\
\hline ht2 & 0.169 & 0.194 & 0.17 & 0.33 & 0.10 \\
\hline ht3 & 0.174 & 0.188 & 0.49 & 0.53 & 0.68 \\
\hline ht4 & 0.151 & 0.150 & 0.79 & 0.40 & 0.08 \\
\hline ht5 & 0.151 & 0.142 & 0.59 & 0.55 & 0.87 \\
\hline
\end{tabular}




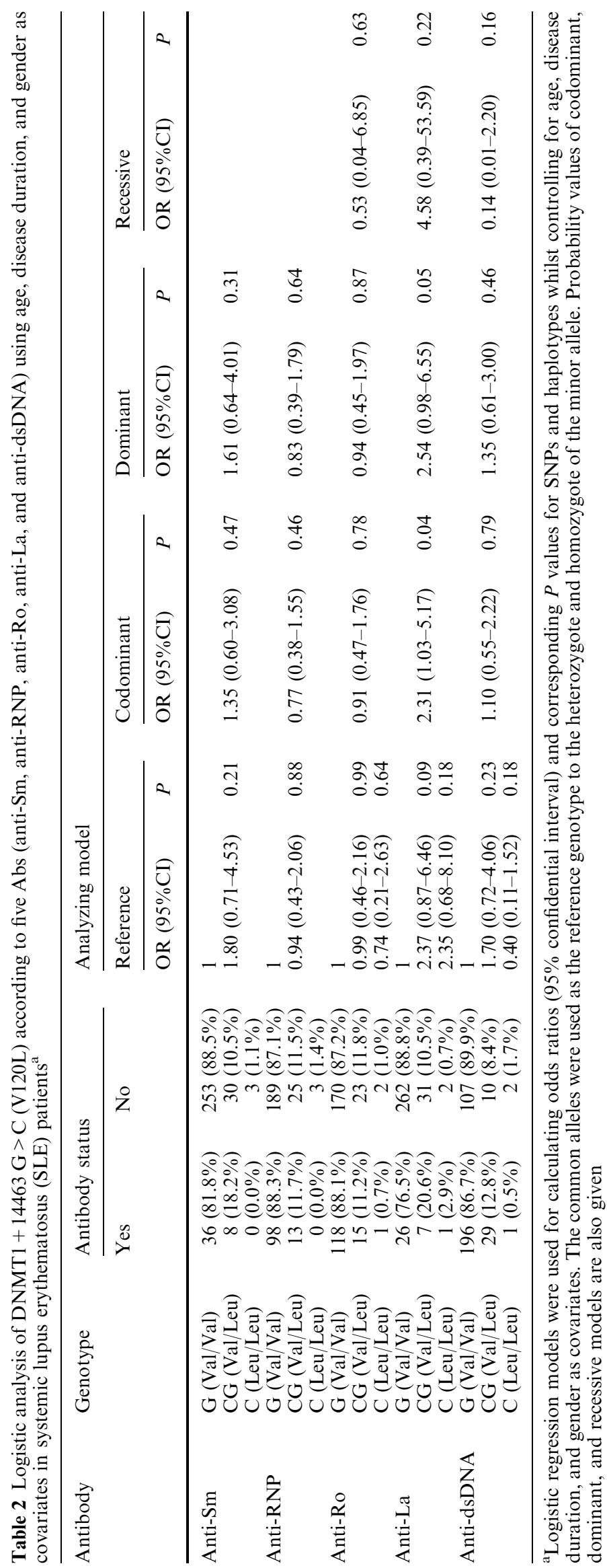


Five major haplotypes accounted for over $87 \%$ of distribution (Fig. 1b).

It had been proposed that genetic factors play a significant role in susceptibility to SLE, in determining the disease expression, and in the autoantibody profiles of individuals with SLE (Wakeland et al. 2001). Recently, it was demonstrated that the pathogenesis of autoimmune diseases such as SLE might be related to DNA methylation and its regulatory enzymes (Okada et al. 2002; Sekigawa et al. 2003). In order to identify the association of DNMT1 polymorphisms with the risk of SLE, logistic regression analyses were performed. The summary in Table 1 demonstrated that no significant associations with the risk of SLE were detected, e.g., no significant differences of allele frequencies were detected between SLE patients and normal controls.

A hallmark of autoimmune diseases such as SLE is the production of highly specific autoantibodies that recognize evolutionarily conserved molecules. SLE is the prototypic nonorgan-specific autoimmune disease in which polyclonal B-cell activation is reflected by a wide range of autoantibody specificities. Previous studies have shown the association of autoantibodies and SLE with various clinical manifestations. Specific autoantibodies accumulate progressively in patients with SLE, even when the patients remain asymptomatic (Arbuckle et al. 2003; Jacob and Viard 1992). DNA methylation and its regulatory enzymes have been suggested to be involved in pathogenesis of autoimmune diseases such as SLE (Okada et al. 2002; Sekigawa et al. 2003). The genetic associations with the production of five autoantibodies (anti-dsDNA, anti-Sm, anti-RNP, anti-Ro, and $\mathrm{La} \mathrm{Abs}$ ) were analyzed with logistic regression models whilst controlling for age, disease duration, and gender as covariates. One nonsynonymous SNP, $+14463 \mathrm{G}>\mathrm{C}(\mathrm{V} 120 \mathrm{~L})$ in exon 4 , was weakly associated with an increased risk of anti-La Ab production among SLE patients $(P=0.04)$ although the significance could not be retained after correction of multiple tests (Table 2). Without further functional evidences, it would be hard to confirm the mechanisms of this association. However, it might be worthwhile to follow up on the association of amino-acid change from Val to Leu with the increased risk of anti- $\mathrm{La} \mathrm{Ab}$ production in larger cohort studies.

In summary, we identified 29 polymorphisms in the human DNMT1 gene. Genetic association analyses with the risk and clinical profiles of SLE revealed no associations with the risk of SLE. However, in further analyses with the production of autoantibodies among SLE patients, one nonsynonymous SNP, $+14463 \mathrm{G}>\mathrm{C}$ (V120L) in exon 4, was weakly associated with an increased risk of anti-La $\mathrm{Ab}$ production among SLE patients although the significance could not be retained after correction of multiple tests (Table 2).

Acknowledgements This work was supported in part by the research fund of Hanyang University, Seoul, Korea (2002-T).

\section{References}

Arbuckle MR, McClain MT, Rubertone MV, Scofield RH, Dennis GJ, James JA, Harley JB (2003) Development of autoantibodies before the clinical onset of systemic lupus erythematosus. N Engl J Med 349:1526-1533

Bestor TH (1992) Activation of mammalian DNA methyltransferase by cleavage of a $\mathrm{Zn}$ binding regulatory domain. EMBO J 11:2611-2617

Chuang LS, Ian HI, Koh TW, Ng HH, Xu G, Li BF (1997) Human DNA-(cytosine-5) methyltransferase-PCNA complex as a target for p21WAF1. Science 277:1996-2000

Gladman D, Ginzler E, Goldsmith C, Fortin P, Liang M, Urowitz M, Bacon P, Bombardieri S, Hanly J, Hay E, Isenberg D, Jones J, Kalunian K, Maddison P, Nived O, Petri M, Richter M, Sanchez-Guerrero J, Snaith M, Sturfelt G, Symmons D, Zoma A (1996) The development and initial validation of the Systemic Lupus International Collaborating Clinics/American College of Rheumatology damage index for systemic lupus erythematosus. Arthritis Rheum 39:363-369

Hedrick PW (1987) Gametic disequilibrium measures: proceed with caution. Genetics 117:331-341

Hedrick P, Kumar S (2001) Mutation and linkage disequilibrium in human mtDNA. Eur J Hum Genet 9:969-972

Hochberg MC (1997) Updating the American College of Rheumatology revised criteria for the classification of systemic lupus erythematosus. Arthritis Rheum 40:1725

Huck S, Deveaud E, Namane A, Zouali M (1999) Abnormal DNA methylation and deoxycytosine-deoxyguanine content in nucleosomes from lymphocytes undergoing apoptosis. Fed Am SocExp Biol J 13:1415-1422

Jacob L, Viard JP (1992) Anti-DNA antibodies and their relationships with anti-histone and anti-nucleosome specificities. Eur J Med 1:425-431

Jones PA, Takai D (2001) The role of DNA methylation in mammalian epigenetics. Science 293:1068-1070

Kanai Y, Ushijima S, Nakanishi Y, Sakamoto M, Hirohashi S (2003) Mutation of the DNA methyltransferase (DNMT) 1 gene in human colorectal cancers. Cancer Lett 192:75-82

Lei H, Oh SP, Okano M, Juttermann R, Goss KA, Jaenisch R, Li E (1996) De novo DNA cytosine methyltransferase activities in mouse embryonic stem cells. Development 122:3195-3205

Li E, Bestor TH, Jaenisch R (1992) Targeted mutation of the DNA methyltransferase gene results in embryonic lethality. Cell 69:915-926

Livak KJ (1999) Allelic discrimination using fluorogenic probes and the $5^{\prime}$ nuclease assay. Genet Anal 14:143-149

Okada M, Ogasawara H, Kaneko H, Hishikawa T, Sekigawa I, Hashimoto H, Maruyama N, Kaneko Y, Yamamoto N (2002) Role of DNA methylation in transcription of human endogenous retrovirus in the pathogenesis of systemic lupus erythematosus. J Rheumatol 29:1678-1682

Okano M, Xie S, Li E (1998) Cloning and characterization of a family of novel mammalian DNA (cytosine-5) methyltransferases. Nat Genet 19:219-220

Richardson B, Scheinbart L, Strahler J, Gross L, Hanash S, Johnson M (1990) Evidence for impaired T cell DNA methylation in systemic lupus erythematosus and rheumatoid arthritis. Arthritis Rheum 33:1665-1673

Robertson KD (2001) DNA methylation, methyltransferases, and cancer. Oncogene 20:3139-3155

Sekigawa I, Okada M, Ogasawara H, Kaneko H, Hishikawa T, Hashimoto H (2003) DNA methylation in systemic lupus erythematosus. Lupus 12:79-85

Stephens M, Smith NJ, Donnelly P (2001) A new statistical method for haplotype reconstruction from population data. Am J Hum Genet 68:978-989

Wakeland EK, Liu K, Graham RR, Behrens TW (2001) Delineating the genetic basis of systemic lupus erythematosus. Immunity 15:397-408 